\title{
Unconventional ferromagnetism and transport properties of (In,Mn)Sb dilute magnetic semiconductor
}

\author{
V. N. Krivoruchko,V. Yu. Tarenkov, D. V. Varyukhin, A. I. D'yachenko \\ Donetsk Physics 83 Technology Institute NAS of Ukraine, \\ Street R. Luxemburg 72, 83114 Donetsk, Ukraine \\ O. N. Pashkova, V. A. Ivanov \\ N. S. Kurnakov Institute of General and Inorganic \\ Chemistry of the Russian Academy of Sciences, \\ 31 Leninsky av., 119991 Moscow, Russia
}

(Dated: December 4, 2018)

\begin{abstract}
Narrow-gap higher mobility semiconducting alloys $\mathrm{In}_{1-x} \mathrm{Mn}_{x} \mathrm{Sb}$ were synthesized in polycrystalline form and their magnetic and transport properties have been investigated. Ferromagnetic response in $\mathrm{In}_{0.98} \mathrm{Mn}_{0.02} \mathrm{Sb}$ was detected by the observation of clear hysteresis loops up to room temperature in direct magnetization measurements. An unconventional (reentrant) magnetization versus temperature behavior has been found. We explained the observed peculiarities within the frameworks of recent models which suggest that a strong temperature dependence of the carrier density is a crucial parameter determining carrier-mediated ferromagnetism of (III,Mn)V semiconductors. The correlation between magnetic states and transport properties of the sample has been discussed. The contact spectroscopy method is used to investigate a band structure of (InMn) $\mathrm{Sb}$ near the Fermi level. Measurements of the degree of charge current spin polarization have been carried out using the point contact Andreev reflection (AR) spectroscopy. The AR data are analyzed by introducing a quasiparticle spectrum broadening, which is likely to be related to magnetic scattering in the contact. The AR spectroscopy data argued that at low temperature the sample is decomposed on metallic ferromagnetic clusters with relatively high spin polarization of charge carriers (up to $65 \%$ at $4.2 \mathrm{~K}$ ) within a cluster.
\end{abstract}




\section{INTRODUCTION}

Carrier-induced ferromagnetic order in Mn-doped III-V alloys attracts much attention at present due to their promising combination of magnetic and semiconducting properties (for reviews on the topic see Refs. [1, 2, 3, 4] ). However, the applicability of these semiconductors in microelectronic technologies requires an increase of their Curie temperature, $\mathrm{T}_{C}$, above room temperature. A progress in the field at present is as large as $\mathrm{T}_{C} \approx 170 \mathrm{~K}$ observed for $\mathrm{Ga}_{1-x} \mathrm{Mn}_{x} \mathrm{As}$ [5]. The issue of whether or not it is realistic to expect for (III,Mn) V materials to have room-temperature $\mathrm{T}_{C}$ is the key question in the field and motivates many theoretical and experimental studies. Ferromagnetism in dilute magnetic semiconductors (DMSs) is usually ascribed to carrier-mediated mechanisms and depends on many different parameters (such as carrier density, magnetic impurity density, coupling between the ion magnetic moment and the hole/electron spin, details of disorder, etc.) which vary greatly from system to system and even from sample to sample. According to current theoretical models [1, 2, 3, 4, 6, 7, ㅇ, 9] stability of ferromagnetic state can be enhanced by increasing carriers density in the vicinity of the magnetic impurities. It was also demonstrated that one can control the strength of ferromagnetic coupling (up to 25\%) by using hydrostatic pressure without any change in the carriers concentration [10].

The picture of the ferromagnetic transition in (III,Mn)V DMSs which seems to be accepted more or less universally is as follows. When $\mathrm{Mn}^{3+}$ substitutes the cation (group III element) in the lattice, the Mn absorbs an electron converting into $\mathrm{Mn}^{2+}$ and produces a hole. Due to strong Hund's interaction, the half-filled $3 \mathrm{~d}^{5}$-shell $\mathrm{Mn}^{2+}$ is spin polarized. On the other hand, the hole experiences a Coulomb attraction to the $\mathrm{Mn}^{2+}$ creating a spin-polarized acceptor level. This hole with ferromagnetically ordered Mn moments form such an object as a bound magnetic polaron, which is a localized carrier, magnetically

strongly correlated with a few neighboring magnetic moments [11]. Since the concentration of magnetic impurities is much larger than the hole concentration, most likely due to compensation by group $\mathrm{V}$ element antisite defects, a bound magnetic polaron consists of one localized hole and a large number of magnetic impurities around the hole localization center. Even though the direct exchange interaction between the localized magnetic impurities is antiferromagnetic, the interaction between bound magnetic polarons may be ferromagnetic at large enough concentrations of holes. The ferromagnetic transition is thought to 
be caused by the temperature-driven magnetic percolation transition of bound magnetic polarons [1, 2, 3, 4, 6, 7, 8, 9, 12]. Typically, the ferromagnetic $\mathrm{T}_{C}$ due to bound magnetic polarons percolation is relatively low. The answer whether or not it is realistic to expect for this class of DMSs to have Curie temperature above room temperature is still a matter of controversy [1, 2, 3, 4].

Recently new arguments have been found which open a possibility to range the Curie temperature in (III,Mn)V DMSs up to room temperature [8, 9]. These models account for the temperature dependence of the carriers which could, in principle, leads to a high-T carriermediated ferromagnetism in semiconductors. In particular, the authors demonstrated the possibility of stable reentrant ferromagnetism. Namely, in contrast to the standard monotonic decay of magnetization with increasing temperature, as the temperature is increased the higher density of thermally exited carriers can enhance the exchange coupling between magnetic ions and thus increase the magnetization over some temperature interval.

As already mentioned, $\mathrm{Mn}^{2+}$ ions substituting for a trivalent cation provide magnetic moment and act as a source of valence-band holes that mediate the $\mathrm{Mn}^{2+}-\mathrm{Mn}^{2+}$ interaction [1, 2, 3, 4, 6, 7, 8]. This interaction results in a ferromagnetic phase with, as expected, half-metallic properties. The key role of spin-polarized charge carriers which mediate ferromagnetic interaction between localized $\mathrm{Mn}^{2+}$ ions, motivates researchers to explore transport properties of the compounds by the point contact (PC) Andreev reflection (AR) techniques. Indeed, during the last years it was demonstrated that AR spectroscopy is an appropriate way for direct measurement of electrical current spin polarization in a variety of materials, including ferromagnetic metals, metallic oxides, half-metals and semiconductors (see, e.g., Refs. [13, 14, 15] and references therein). The method is based on the difference in the AR in normal metal/superconductor $(\mathrm{N} / \mathrm{S})$ and in ferromagnet/superconductor $(\mathrm{F} / \mathrm{S})$ contacts [16]. However, it is not an easy task to determine the electrical current spin polarization in DMSs by the AR spectroscopy. The Schottky barrier fundamentally limits the accuracy of spin-polarization measurements strongly decreasing the probability of the AR. To avoid this problem, one should use heavy doped semiconductors with metallic-type conductivity. Fortunately, for higher mobility ferromagnetic semiconductors, such as $\operatorname{In}_{1-x} \mathrm{Mn}_{x} \mathrm{Sb}$, the Schottky barrier is thin, which makes the $\mathrm{S} / \mathrm{In}_{1-x} \mathrm{Mn}_{x} \mathrm{Sb}$ interface highly transparent and thus the AR methods applicable [17, 18, 19].

An interesting result of experimental efforts in the field, which is also important for 
developing spin based devices, is discovery of a large magnitude of charge-carriers (holes) spin polarization in filmy samples of Mn-doped III-V semiconductors. In particular, the $\mathrm{Ga}_{1-x} \mathrm{Mn}_{x}$ As is one of the thoroughly characterized DMS and for this alloy the AR measurements have revealed a magnitude of the carrier spin polarization up to $\approx 85 \%$ for samples with 5-8\% Mn [20, 21]. Such an alloy as (In,Mn)Sb is another relatively new ferromagnetic material, which has the largest lattice constant in the family of (III,Mn)V materials. Since (In,Mn)Sb has the smallest effective mass of the holes, it has much higher hole mobility than other (III,Mn)V ferromagnetic semiconductors. It is also unique among the ferromagnetic (III,Mn)V family due to the smallest energy gap. For $\operatorname{In}_{1-x} \mathrm{Mn}_{x} \mathrm{Sb}$ filmy samples chargecarriers spin polarization was found to be as large as $52 \%$ at liquid helium temperature [18].

In this communication, we report on the fabrication of the semiconducting alloys $\mathrm{In}_{1-x} \mathrm{Mn}_{x} \mathrm{Sb}(\mathrm{x}=0$ and $2 \% \mathrm{Mn})$ in the form of large-grained polycrystals and the results of the samples' macroscopic magnetic and transport properties studies. Direct measurements of magnetization in $\mathrm{In}_{0.98} \mathrm{Mn}_{0.02} \mathrm{Sb}$ were carried out and ferromagnetic order is unambiguously established by the observation of the magnetization hysteresis loops up to room temperature. We also observed an unconventional magnetization $v s$ temperature behavior: the magnitude of the magnetization is increased by about $3 \%$ as temperature increase from $4.2 \mathrm{~K}$ to room temperature. We explain the results found based on the models of carrier-mediated ferromagnetism in (III,Mn)V DMSs which account for the temperature dependence of the carriers density. The correlation between magnetic states and transport properties of the sample has been examined. Particularly, the point contact Andreev reflection spectroscopy has been used to investigate uniformity of a low-temperature magnetic order and charge carriers spin polarization in the DMS under consideration. For some of the junctions the measured AR spectrum displays characteristics which are typical for PCs of a BCS superconductor with a high current spin polarized conductor. For these PCs the charge-carriers spin polarization was found to be as large as $65 \%$ at liquid helium temperature. Apart form AR spectroscopy we also performed tunnel spectroscopy studies and investigated a band structure of (InMn)Sb near the Fermi level.

The paper is organized as follows. In Section II the experimental details and some general properties of the samples are presented. Section III is devoted to the magnetostatic data obtained. Discussion of these experimental results and its comparison with models 
predictions one can find in Sec.IV. The data for bulk transport properties of the samples are given in Sec. V. Here a correlation between the bulk magnetic state and transport properties of the sample has been expected, including the possibility of a simultaneous magnetic and transport percolation [22, 23, 24]. The experimental results on Andreev reflection spectroscopy and related discussion one can find in Sec. VI. We end with the Summary.

\section{SAMPLES AND EXPERIMENTAL DETAILS}

A basic material, InSb, has the largest unit sell and narrowest band gap $(0.17 \div 0.27 \mathrm{eV})$ among III-V semiconductors. Lightly doped InSb-Mn has also the shallowest acceptor level among Mn-doped III-V DMSs [2, 26]. However, the equilibrium solubility of Mn in III$\mathrm{V}$ compounds is quite low $\left(\sim 10^{19}\right.$ per $\left.\mathrm{cm}^{3}\right)$. Bulk samples of the solid solutions InSbMn (2 at.\% Mn) were synthesized in Institute of General and Inorganic Chemistry RAS (Moscow, Russia) as described in details earlier [27]. In brief, the starting materials were single crystal ISE-2 'v' n-type InSb (with electron concentration $2 \times 10^{14} \mathrm{~cm}^{-3}$ at $77 \mathrm{~K}$ and mobility $\left.5 \times 10^{5} \mathrm{~cm}^{2} /(\mathrm{V} \mathrm{s})\right)$ and $99.9 \%$ pure $\mathrm{Mn}$ and $\mathrm{Sb}$. The polycrystalline solid solutions $\mathrm{In}_{1-x} \mathrm{Mn}_{x} \mathrm{Sb}$ were synthesized in evacuated quartz ampoules under isothermal conditions throughout the length of the ampoule. The samples have been melted at $800^{\circ} \mathrm{C}$ for $2 \mathrm{~h}$, followed by cooling to $550^{\circ} \mathrm{C}$ and solidification under nonequilibrium conditions. The crystallographic quality of the $\mathrm{In}_{1-x} \mathrm{Mn}_{x} \mathrm{Sb}$ specimen was studied using Fe $K \alpha$ radiation. The measured lattice constant, $a=6.478 \AA$, coincides with that of InSb within the accuracy of the analysis. None of the samples studied show any significant impurity peaks corresponding to such unwanted phases like Mn complexes or MnSb.

To examine quantitatively the $\mathrm{Mn}^{2+}$ distribution, the chemical composition analysis of the $\mathrm{In}_{0.98} \mathrm{Mn}_{0.02} \mathrm{Sb}$ sample was carried out by X-ray energy dispersive spectroscopy (EDS) using INCAPentaFETx3 spectrometer and JSM-6490LV scanning electron microscopy (SEM). The spatial resolution of the spectrometer in a single step is about 100nm. The crystal's fresh chip micrograph is shown in Fig. 1. The elemental distribution of the sample was checked using the point and shoot microanalysis at various points. Representative data obtained from the EDS spectra for eleven points in Fig. 1 are summarized in Table I. As it follows from the EDS data, the Sb concentration varies slightly; the concentrations of In and Mn mutually 
correlate. We attribute detection of the $\mathrm{O}$ ions to adsorption of oxygen on the sample's surface from air. So, we suggested that all Mn ions are in a solid alloy state, though this alloy is somewhat inhomogeneous. Within an accuracy of the spectrometer resolution, we did not find a decomposition of the sample into regions with large and small concentration of magnetic ions. As it follows from the results obtained, the average concentration of $\mathrm{Mn}^{2+}$ ions is $1.96(0.8) \%$.

According to previous investigations [27], the $\operatorname{In}_{1-x} \mathrm{Mn}_{x} \mathrm{Sb}$ solid solutions with $\mathrm{x} \geq 1 \% \mathrm{Mn}$ are $p$-type with a room temperature hole concentration of $\sim 10^{19} \mathrm{~cm}^{-3}$. This means that manganese doping of $n$-type InSb caused a conversion to $p$-type and increased the carrier concentration by four orders of magnitude in comparison with the pure compound semiconductor InSb (see also comments to Fig. 7 below). It is worth to note, that the carrier concentration in the synthesized materials is an order of magnitude greater than the highest carrier concentration, $\sim 10^{18} \mathrm{~cm}^{-3}$, in $\operatorname{In}_{1-x} \mathrm{Mn}_{x} \mathrm{Sb}$ single crystals grown by Czochralski technique under near-equilibrium conditions and an order of magnitude lower than the manganese concentration, $\sim 10^{20} \mathrm{~cm}^{-3}$, due to compensating defects.

Magnetic properties of the samples were measured by PPMS- 9 system (Quantum Design) operated in temperature range from $4.2 \mathrm{~K}$ to $350 \mathrm{~K}$ under an applied magnetic field up to $9 \mathrm{~T}$. The current-voltage (I vs $\mathrm{V}$ ) characteristics were measured using a conventional four-probe method. Resistivity as a function of temperature was measured directly by using an $a c$ voltage bias source with a small output resistance and $\sim 400 \mu \mathrm{V}$ amplitude of signal on the sample. The tunneling measurements were carried out on tunnel junctions formed by pressing a small Ag piece (counterelectrode) against the sample. Tunnel barrier is formed by the native oxide surface on Ag. For the AR spectroscopy, low-temperature superconductor $\mathrm{Pb}$ is used as a superconducting electrode. Metallic contacts between $\mathrm{In}_{0.98} \mathrm{Mn}_{0.02} \mathrm{Sb}$ plate and superconducting wire were formed by pressing slide-squash up a needle-shaped superconductor by a micrometric screw against the mechanically polished plate's surface. To record the AR spectra $(\mathrm{dI} / \mathrm{dV}$ vs $\mathrm{V}$ ) of the point contacts, we used $150 \div 200 \mu \mathrm{V}$ modulating $a c$ voltage. The resistance of the current and potential electrodes was $\mathrm{R} \sim 10^{-4}$ $\Omega \times \mathrm{cm}^{2}$. The junctions resistance was much larger $(\sim 1 \div 5 \Omega)$, so that the rescaling effects can be neglected. The contacts' parameters were stable, offering a possibility to perform measurements in wide temperature range.

In the report, the results obtained on ferromagnetic $\operatorname{In}_{1-x} \mathrm{Mn}_{x} \mathrm{Sb}$ with $\mathrm{x}=0.02$ are com- 
pared where it is possible with the similar data for ferromagnetic MnSb and nonmagnetic $\mathrm{InSb}$.

\section{MAGNETIC PROPERTIES: EXPERIMENT}

In Fig. 2, magnetization $\mathrm{M}(\mathrm{H})$ of $\mathrm{In}_{0.98} \mathrm{Mn}_{0.02} \mathrm{Sb}$ (main panel) and, for comparison, of MnSb (bottom inset) prepared under the same conditions are shown. Surprisingly, for $\mathrm{In}_{0.98} \mathrm{Mn}_{0.02} \mathrm{Sb}$, clear hysteresis loops are detected not only at $\mathrm{T}=5 \mathrm{~K}$ or $77 \mathrm{~K}$, but up to $300 \mathrm{~K}$ (the highest upper temperature available in the experiment). One can see in the figure that the $\mathrm{M}(\mathrm{H})$ saturates at fields $\approx 4 \mathrm{kOe}$ at $\mathrm{T}=5 \mathrm{~K}$. The dependence of magnetization on temperature in magnetic field $\mathrm{H}=1 \mathrm{kOe}$ is shown in upper inset. The data revealed that the sample of $\mathrm{In}_{0.98} \mathrm{Mn}_{0.02} \mathrm{Sb}$ is ferromagnetic with relatively small coercivity (anisotropy) up to room temperature, and that the saturation magnetization is $\approx 1.45 \mathrm{emu} / \mathrm{g}$. The main result here shown in the upper inset is that the magnitude of the magnetization is increased by about 3\% as temperature increases from 4.2K to room temperature.

It should be noted here, that there are various experimental difficulties in the unambiguous determination of the origin of magnetic signals in the DMS containing a minute number of magnetic ions. A main question here is whether a spatially uniform ferromagnetic order is a real ground state in this DMS or not? When the concentration of magnetic impurities exceeds the solubility limit, a variety of magnetic nanocrystals embedded into semiconductor may be formed. In our case, as a first possibility, one can argue that the detected hysteresis loops may be due to formation of MnSb clusters with $\mathrm{T}_{C} \approx 580 \mathrm{~K}$. For the $\operatorname{In}_{1-x} \mathrm{Mn}_{x} \mathrm{Sb}_{\mathrm{sam}}$ ples with $\mathrm{x}=1 \div 1.3 \% \mathrm{Mn}$ prepared by the same group, such suggestion was earlier invented in Ref. [28]. Indeed, the equilibrium solubility of Mn in III-V compounds is quite low and, if the Mn concentration is beyond the solubility limit, one should expect a formation of nano-regions with different concentration of magnetic ions. However, for the $\operatorname{In}_{0.98} \mathrm{Mn}_{0.02} \mathrm{Sb}$ sample, with increasing temperature, we detected small but visible increase of the magnetization (see upper inset in Fig. 2) and increase of the coercivity field. The behavior of both is untypical for ferromagnets. In addition, for $\mathrm{MnSb}$, at the same conditions, $\mathrm{T}=$ $300 \mathrm{~K}$, we were not able to detect the coercivity field: the $\mathrm{M}(\mathrm{T})$ curve is quite reversible (see bottom inset in Fig. 2). We also tried to fit the $\mathrm{M}(\mathrm{H}, \mathrm{T})$ data for the $\operatorname{In}_{0.98} \mathrm{Mn}_{0.02} \mathrm{Sb}$ sample at $\mathrm{T}=4.2 \mathrm{~K}$ and $300 \mathrm{~K}$ by the Langevin function and to restore a typical size of possible 
ferromagnetic clusters. However, we found that this is impossible if one assumes that the size of a cluster is fixed.

For some of the cases, ferromagnetic response may be due to formation of Mn-rich nanoclusters (a spinodal decomposition) [4, 29]. It is well known that phase diagrams of a number of alloys exhibit a solubility gap in a certain concentration range. This may lead to a spinodal decomposition into regions with a low and a high concentration of magnetic constituent. It may appear in a form of coherent nanocrystals embedded by the majority component. Since spinodal decomposition does not usually involve a precipitation of another crystallographic phase, it is not easily detectable experimentally. Nevertheless, its presence was found in (Ga,Mn)As, where coherent zinc-blende Mn-rich (Mn,Ga)As metallic nanocrystals led to an apparent Curie temperature up to $360 \mathrm{~K}$ [29]. As was found, the magnetization behavior for Mn-rich clusters is a conventional one (see, e.g., Fig.5b in Ref.[29]) while we observe unconventional M(T) behavior. Still, a possibility that a small number of $\mathrm{MnSb}$ or Mn-reach nanoclusters are formed in (InMn)Sb matrix cannot be ruled out completely.

It may be also argued that the samples are polycrystalline and ferromagnetism could be due to a surface. Indeed, room temperature ferromagnetism has been observed in a variety of inorganic nanoparticles although the materials are intrinsically non-magnetic [30]. The point defects at the surface are likely to be responsible for this phenomenon. However, the appearance of ferromagnetism is detected only for small enough particles $(<10 \mathrm{~nm})$, while it disappears in the bigger particles [30]. For our polycrystalline samples the ratio surface/volume is very small which casts doubts on the origin of magnetism observed due to surface imperfections. Yet, the role of grain boundaries remains open and experiments with another samples of different Mn content could shed light on question whether the observed reentrant ferromagnetism is due to polycrystallinity or not.

In Fig. 3, we compare our data with those of Ref. [18]. In the figure open circles are the magnetic field dependence of magnetization in $\operatorname{In}_{1-x} \mathrm{Mn}_{x} \mathrm{Sb}(\mathrm{x}=0.028)$ epitaxial film (with $\mathrm{T}_{C} \approx 9 \mathrm{~K}$ ) at $\mathrm{T}=50 \mathrm{~K}[18]$. One can see that this data is very well correlated with ours (compare the results of Ref. [18] at $\mathrm{T}=50 \mathrm{~K}$ and our data at $\mathrm{T}=77 \mathrm{~K}$ ). So, we can suggest that an unconventional $\mathrm{M}(\mathrm{T})$ dependence may be observed in film samples too. The occurrence of robust ferromagnetism at room temperature in bulk (InMn)Sb semiconductors with $\mathrm{Mn}$ up to $1.33 \%$ was reported in Refs. [28, 31]. However, the authors were not able 
to determine whether the magnetism is truly a bulk phenomenon. The observation also remained unexplained. In our opinion, the observed $\mathrm{M}(\mathrm{T})$ behavior of $\mathrm{In}_{0.98} \mathrm{Mn}_{0.02} \mathrm{Sb}$ can be understood along the lines of the recently proposed models [8, 9]. This point is further discussed below. It is worth to note here that an unconventional $\mathrm{M}(\mathrm{T})$ behavior has already been observed in some other DMSs [32]. These observations were explained based on quite different approaches.

\section{MAGNETIC PROPERTIES: DISCUSSION}

We apply the model [8] to describe qualitatively the data in Figs. 2 and 3. As was already mentioned, a $\mathrm{Mn}$ impurity in $\operatorname{In}_{1-x} \mathrm{Mn}_{x} \mathrm{Sb}$ is presented by two spin-degenerate levels: 'the deep level' and 'the shallow level'. The deep level, when occupied by one electron, provides the impurity's spin $(S=5 / 2)$. The shallow level plays the role of impurity's acceptor level. The shallow, acceptor levels of the impurities are to donate, on average, less then one hole per impurity. So, the Fermi level, $\varepsilon_{F}$, coincides with the energy of a hole placed onto the shallow level of an impurity (see Fig. 4).

Let us consider a hole associated with a particular $\mathrm{Mn}^{2+}$ ion which is confined in hydrogenlike orbital of radius $r_{H}$. As the acceptor concentration increases, the hydrogen-like orbitals $\psi(r)=\left(\pi r_{H}^{3}\right)^{-1 / 2} \exp \left(-r / r_{H}\right)$ overlap to form an impurity band. At first, the holes remain localized because of the influence of correlations and the donors tend to form bound magnetic polarons [7, 8, 10]. If the radius $\mathrm{r}_{H}$ is sufficiently large, overlap between a hydrogen hole and the cations within its orbit leads to ferromagnetic exchange coupling between them [33]. This interaction may be written in terms of the $p$ - $d$ exchange parameter $\mathrm{J}_{p d}$ as: $\sum_{i} \mathrm{~J}_{p d} \mathbf{S}_{i} \mathbf{s}\left(\mathbf{R}_{i}\right)$, where $\mathbf{S}_{i}$ is the impurity spin located at $\mathbf{R}_{i}, \mathbf{s}\left(\mathbf{R}_{i}\right)$ is the carrier spin density at $\mathbf{R}_{i}$. (Following the main ideas of the model [8] we still consider a simplified case by taking into account only a long-range field from delocalized holes and by ignoring a short-range field acting on the impurity spin from bound electrons.) Since the concentration of holes is much smaller than that of impurities, one localized hole is surrounded by many magnetic impurities. Exchange interaction between the hole and magnetic impurities leads to their mutual polarization when temperature is below exchange constant $\mathrm{J}_{p d}$. This structure, which consists of an almost completely spin-polarized hole and magnetic impurities polarized by it, is conventionally called 'bound magnetic polaron' [11, 12, 33]. As the density of polarons 
increases, the polarons overlap forming polaron cluster with all impurities within this cluster having their spins aligned in the same direction. Ferromagnetism occurs when the polaron percolation threshold is overlapped and the infinite clusters spanning whole sample appears. The option for boosting $\mathrm{T}_{C}$ significantly is somehow to increase the hole's density in the vicinity of the magnetic impurity. Indeed, within mean-field approximation, the impurity spins act upon the carrier spins as an effective magnetic field $\propto J_{p d} N_{i} m(T)$; while the hole spins act upon the impurity spin with an effective field $\propto J_{p d} N_{h} \nu(s, T) s(T)$. Here $\mathrm{N}_{i}$ is density of magnetic impurities and $\mathrm{N}_{h}$ is hole's density, $\nu(s, T)$ is temperature dependent relative density of holes $\nu(s, T)=n(s, T) / N_{h}$ where $n(s, T)$ is the holes density. As the result, the magnetization of the magnetic impurities is described by

$$
m(T)=J B_{J}\left[J \frac{J_{p d} N_{h} \nu(s, T)}{k_{B} T} s(T)\right]
$$

where $B_{J}(x)$ denotes the Brillouin function, while the magnetization of conduction holes is

$$
s(T)=\frac{1}{2} \tanh \left[\frac{J_{p d} N_{i}}{2 k_{B} T} m(T)\right]
$$

By using Eqs. (1) and (2) one can obtain an expression for the Curie temperature [8]:

$$
T_{C}=J_{p d}\left[N_{i} N_{h} J(J+1) / 12\right]^{1 / 2} \nu\left(0, T_{C}\right)=T_{C}^{0} \nu\left(0, T_{C}\right)
$$

where $T_{C}^{0}$ is the Curie temperature in the limit of completely ionized donors [34]. As it follows form Eq. (3), due to the factor $\nu(s, T)$, an increase number of thermally excited carriers may be sufficient to increase the exchange coupling between magnetic impurities, that is, a ferromagnetic state may appear at higher temperature.

To describe the magnetization dependence on temperature, we assume the valence band is separated from impurity acceptor levels by energy $\varepsilon_{d}$ in the absence of magnetic order (see Fig. 4). For holes' relative density dependence on temperature the expression:

$$
n(s, T)=\frac{1}{2} N_{h} \kappa(s, T)[\sqrt{1+4 / \kappa(s, T)}-1],
$$

is adopted [8] where the function $\kappa(s, T)$ is $\kappa(s, T)=N_{c} / N_{h} \exp \left(-\varepsilon_{d} / k_{B} T\right)$, and $N_{c}=$ $2.5\left(\mathrm{~m}^{*} / \mathrm{m}_{0}\right)^{3 / 2}(\mathrm{~T} / 300 \mathrm{~K})^{3 / 2} \times 10^{19} \mathrm{~cm}^{-3}[35]$. Materials parameters were chosen from the data found in the literature for $\operatorname{In}_{1-x} \mathrm{Mn}_{x} \mathrm{Sb}$ alloys [26, 27]. For numerical simulation, we suggested that $N_{h} \approx 1.1 \times 10^{19} \mathrm{~cm}^{-3}, N_{i} \approx 8.8 \times 10^{20} \mathrm{~cm}^{-3}, J_{p d} \approx 200 \div 210 \mathrm{eV} \cdot \AA^{3},\left|\varepsilon_{d}\right| \approx 15.5 \div$ 16.5meV $, J=5 / 2, m^{*} \approx 0.25 \div 0.43 m_{0}$. Fig. 5 illustrates (main panel) the temperature 
dependencies of the average magnetization (per impurity atom) $M(T)=m(T)+\frac{N_{h}}{N_{i}} s(T)$ as well as (inset) the $m(T)$ and $s(T)$ behavior which we obtain from Eqs. (1) and (2). If at $\mathrm{T} \longrightarrow 0$ there is a small but finite carriers concentration (for the data shown in Fig. 5 we chose $\nu(s, T \longrightarrow 0)=0.001)$, there are three different solution for $\mathrm{T}_{C}$ : at low temperature there is ferromagnetic order up to $\mathrm{T}_{C 1}$ while the reentrant ferromagnetic state is stable in the range $\mathrm{T}_{C 2} \leq \mathrm{T} \leq \mathrm{T}_{C 3}$. If at $\mathrm{T}=0$ there are no carries, ferromagnetic state exists only in the range $\mathrm{T}_{C 2} \leq \mathrm{T} \leq \mathrm{T}_{C 3}$ and is absent at both low and high temperatures, corresponding to clustered (or spin-glass) and paramagnetic states, respectively.

It is noteworthy that for (III,Mn)V DMS spin-glass-like state may be an unusual one. In general, there are two possibilities. As was already mentioned, from our present results we cannot rule out completely a probability that a small number of MnSb or Mn-rich nanoclusters are formed in (In,Mn)Sb matrix with mutual off-orientation of clusters' magnetization. Another origin of a spin-glass state is 'frozen' bound magnetic polarons. As is well known (see, e.g., Ref. [36]) for system in a spin-glass state in some of (low) temperature interval one can observe a reentrant magnetization behavior. Taking into account transport properties (see next section), we think that in temperature range $4.2 \mathrm{~K}-30 \mathrm{~K}$ the $\mathrm{In}_{0.98} \mathrm{Mn}_{0.02} \mathrm{Sb}$ sample is in a spin-glass state. In both cases (clustered or spin-glass states) the temperature dependence of the magnetization reveals a nonmonotonic behavior which is very different from the conventional monotonic decay. Returning to Fig. 5, the curves look more exaggerated than the experimental ones, but one should take into account that (i) we have about two Mn ions on hundred unit cells, and (ii) a noticeable part of Mn ions located outside the ferromagnetically ordered regions [23, 24, 25] (see discussion in Secs. VI and V) and are not involved into the machanism under consideration.

The mechanism described is also able to explain, at least qualitatively, our observation of coercivity field increasing. Indeed, while the origin of magnetic anisotropy in DMSs still remains not well understood (see recent publications on the topic [37, 38] and references therein), the experimental results yield for Mn-doped DMS the linear dependence of the uniaxial anisotropy to be proportional to the holes concentration [37]. So, the thermal activation of carriers may enhance as the exchange coupling between magnetic impurities as well as the magnetic anisotropy. Unfortunately, values of the basic parameters of the model, $J_{p d}$ and $\varepsilon_{d}$, are not known exactly at present, and their scatter leads to both low and room $\mathrm{T}_{C}$ values. 
The scenario above assumed the conduction band is empty. Actually, the carriers may be thermally activated from the valence to the conduction band as well. For mediated by thermally excited free carriers, the effective magnetic interaction is suggested to be of the Ruderman-Kittel-Kasuya-Yosida (RKKY) - type [39]. Since the carrier density is low, the first node of the oscillating RKKY function occurs at a length scale larger than the average Mn-Mn distance, giving rise to a net ferromagnetic interaction (for details see Ref.[6]). Typically the Curie temperature due to the RKKY mechanism is relatively high. As was pointed recently by Calderón and Das Sarma [9], in some DMSs ferromagnetism may appear due to both the exchange coupling of a (localized) carrier in the impurity band with a few neighboring magnetic impurities (the bound magnetic polarons percolation mechanism) and indirect exchange induced by thermally activated carries in an otherwise empty conduction band (the so-called 'activated' RKKY mechanism). In this case, in a sample thermal activation leads to a high-T ferromagnetism due to the RKKY free-carrier mechanism, and to a low-T ferromagnetism due to localized bound carriers in an impurity band through the polaron percolation mechanism. Based on our data we cannot rule this possibility out.

\section{TRANSPORT PROPERTIES}

In Fig. 6 low temperature zero-field resistivity for ferromagnetic $\operatorname{In}_{0.98} \mathrm{Mn}_{0.02} \mathrm{Sb}$ sample is shown. For comparison, in the inset, the temperature dependence (up to room temperature) of resistivity for the $\mathrm{In}_{0.98} \mathrm{Mn}_{0.02} \mathrm{Sb}$ and InSb prepared under the same conditions is also displayed. As one can see in the inset of Fig. 6, addition of Mn ions results in changing the transport properties from semiconducting to 'weakly metallic'. Note that here the term 'metallic' we refer to the scope of the resistivity vs temperature and the term 'weakly' used in the sense that the resistivity does not exhibit strong temperature dependence. At liquid nitrogen temperature the resistivity is $\rho_{(\operatorname{InMn}) S b}(77 \mathrm{~K})=4.2 \times 10^{-4} \Omega \cdot \mathrm{cm}$, corresponding to a metallic mean free path of $k_{F} l \sim 50$.

For system with reentrant ferromagnetic behavior, one can expect that different character of the magnetic order at low-, $\mathrm{T} \leq \mathrm{T}_{C 2}$, and at high-, $\mathrm{T}_{C 2} \leq \mathrm{T} \leq \mathrm{T}_{C 3}$, temperatures will be revealed in a different system's transport properties, too. Indeed, according to Monte Carlo simulations (see, e.g. [23, 24]), in sample containing a few percent of Mn ions, small ferromagnetic regions (clusters) begin to develop even above the mean-field Curie tempera- 
ture where the local Mn density exceeds the average. As T decreases these regions increase and may form a percolation transport network (i.e., metallic conductivity) at $\mathrm{T}_{C 1}$, if ferromagnetic state is realized below $\mathrm{T}_{C 1}$, or the system remains in a clustered/spin-glass state (with activated character of conductivity) down to $\mathrm{T}=0$. In both cases, a fraction of the spins still remain outside the network/clusters providing charge carrier magnetic scattering [23, 24, 25]. Figure 6 shows that in temperature interval from $30 \mathrm{~K}$ to $300 \mathrm{~K}$ the resistance increases with increasing temperature. Careful inspection of the data in Fig. 6 (main panel) reveals that at low temperature $4.2 \mathrm{~K}<\mathrm{T}<30 \mathrm{~K}$ the data are too noisy. So that, we cannot, unfortunately, indisputably conclude whether the system has to have activated-like conductivity or metallic one. A monotonic increase of the magnetization observed is in favor of a spin-glass state [36]. In the next section, we will analyze this temperature region more carefully by the AR spectroscopy.

Above $\mathrm{T}_{C 1}$, as temperature is increased the metallic sate may be realized due to thermally exited carries. In the metallic ferromagnetic phase, $\mathrm{T}_{C 2}<\mathrm{T}<\mathrm{T}_{C 3}$, hole mobility may be explained on the basis of a simple band-transport picture, which facilitates the ideal limit of spin-polarized transport. Typical temperature dependent processes giving rise to resistivity are the electron-electron scattering, $\sim T^{2}$, and magnetic scattering on $\mathrm{Mn}^{2+}$ ions, (for spinpolarized carriers the one-magnon scattering process is forbidden and two-magnon scattering gives $\sim T^{9 / 2}[40]$ ). In accordance with this picture, from the data in Fig. 6, one can find that at $\mathrm{T}>30 \mathrm{~K}$ the resistivity indeed slowly increases as temperature increases up to the highest of measured temperature 300K. Again, because the carrier density is low the temperature behavior is 'weakly metallic' in the sense that the resistivity does not exhibit strong temperature dependence.

Tunneling conductance $\mathrm{G}(\mathrm{V})=\mathrm{dI} / \mathrm{dV}$ for a sample - insulator $(\mathrm{I})$ - tip tunnel junction is a direct method for measuring of the single particle density of state (DOS) of the sample at energy near the Fermi level $\varepsilon_{F}$ [41]. To investigate the changes in DOS due to doping, we have performed electron - tunneling experiments on $\mathrm{InSb}-\mathrm{I}-\mathrm{Ag}$ and $\mathrm{In}_{0.98} \mathrm{Mn}_{0.02} \mathrm{Sb}-\mathrm{I}-$ Ag tunnel junctions. Representative tunnel spectra obtained are shown in Fig. 7. The conductance of the InSb- I- $\mathrm{Ag}$ contacts demonstrates a well-defined forbidden gap in the DOS between the valence and conductivity bands. The width of this gap $\mathrm{W}=0.27 \mathrm{eV}$ is in good accordance with the gap which has been restored earlier from electric measurements [42]. For diluted system we detected two main differences: (i) the forbidden gap is absent 
and (ii) the chemical potential is now shifted corresponding to holes type of conductivity (positive sign of voltage). These features are well reproduced and are detected on large number of $\mathrm{In}_{0.98} \mathrm{Mn}_{0.02} \mathrm{Sb}-\mathrm{I}-\mathrm{Ag}$ tunnel junctions. Taking into account our EDS analyzes, we can with confidence conclude that the main part of Mn ions are a solid solution state and form $\mathrm{In}_{0.98} \mathrm{Mn}_{0.02} \mathrm{Sb}$ matrix unique properties.

\section{ANDREEV REFLECTION SPECTRA}

Experiment. - To study transport properties of ( $\mathrm{In}, \mathrm{Mn}) \mathrm{Sb}$ semiconductor in a low temperature region $\left(\mathrm{T}<\mathrm{T}_{C 2}\right)$, we have prepared and measured a number of different PCs. Below we present a set of data obtained on various samples by contact formation at different positions on the sample surface. Fig. 8 exemplarily shows the representative characteristics of the $\mathrm{Pb}-\mathrm{In}_{0.98} \mathrm{Mn}_{0.02} \mathrm{Sb}$ contact. Here, the $\mathrm{I}-\mathrm{V}$ dependence is presented in the main panel; the top inset exhibits the temperature dependence of the contact's resistance $R(T)$, and the bottom inset illustrates the contact's $A R$ spectra $G(V)$ at $T=4.2 \mathrm{~K}$. As one can see, at $T$ $<\mathrm{T}_{S C}(\mathrm{~Pb})=7.2 \mathrm{~K}$ a sharp drop of the contact's resistivity is detected. Reduction of the resistance for the contact under consideration is about $50 \%$, a theoretical limit which one can expect for a perfect $\mathrm{S} / \mathrm{N}$ junction, and an excess current $\mathrm{I}_{e x c}$ is definitely observed. The current-voltage relation above the superconducting transition temperature is linear, providing additional support for the absence of the Schottky barrier. Any features typical for a $\mathrm{PC}$ of a singlet pairing superconductor with spin-polarized metal [13, 14, 15] have not been detected.

However, for a few exclusive cases we had a chance to detect the PC characteristics which we attributed to (nanoscale) ferromagnetic clusters with spin-polarized charge carriers. In Fig. 9, the representative characteristics of such $\mathrm{Pb}-\mathrm{In}_{0.98} \mathrm{Mn}_{0.02} \mathrm{Sb} \mathrm{PC}$ are exposed. As earlier, the $\mathrm{I}-\mathrm{V}$ dependence is shown in the main panel; the top inset exhibits the temperature dependence of the contact's resistance $R(T)$; and the bottom inset illustrates the contact's AR spectra at $\mathrm{T}=4.2 \mathrm{~K}$. As one can see, in contrast to the case in Fig. 8, at $\mathrm{T}<7.2 \mathrm{~K}$ a sharp growth of the contact's resistivity is observed. Also, again in contrast to the case in Fig. 8, now an excess voltage $\mathrm{V}_{\text {exc }}$ is observed. The almost constant $\mathrm{V}_{\text {exc }}$ value is detected for $|\mathrm{V}| \leq 40 \mathrm{mV}$. This proves the suggestion that heating effects could be neglected. For comparison, we also prepared and measured (not shown) the AR spectra for 
nonmagnetic InSb (the InSb - Pb junctions). For all of the PCs of the 'parent matrix' with $\mathrm{Pb}$ any peculiarities which may be attributed as due to spin-polarized current have not been detected.

Modeling. - As already mentioned, the AR experiments provide a direct measure of the current spin polarization, $P=\left(\mathrm{j}_{\uparrow}-\mathrm{j}_{\downarrow}\right) / \mathrm{j}_{\text {tot }}$, where $\mathrm{j}_{\uparrow(\downarrow)}$ is a partial current of carriers with spin 'up' ('down') and $\mathrm{j}_{\text {tot }}=\left(\mathrm{j}_{\uparrow}+\mathrm{j}_{\downarrow}\right)$. Indeed, the current through a ferromagnet - superconductor interface is determined by the charge conversion of a Cooper pair into individual electrons. As a Cooper pair consists of two electrons with opposite spins, the conversion is suppressed if there is spin discrimination of the bands. So that $\mathrm{P}$ can be deduced from the voltage dependence of the conductance.

The spectra obtained were fitted using the models [13, 14, 15]. The diameter, $d$, of a point contact with resistance $\mathrm{R}_{N}$ was determined for Sharvin-Wexler formula [43]:

$$
R_{N}=\frac{4}{3} \pi d^{2} \rho \ell+\frac{\rho}{2 d}
$$

Here $\ell$ is the mean free path of the charge carriers, and $\rho$ is the bulk resistivity of the material. For $\rho(\mathrm{T}=4.2 \mathrm{~K}) \sim 10^{-4} \Omega \mathrm{cm}$ and a junction's normal state resistance $\mathrm{R}_{N} \sim$ $1 \div 3 \Omega$ the range of $\mathrm{PC}$ diameter values is $\mathrm{d} \geq 300 \AA$; we took for the mean free path its typical value $\ell \sim 100 \AA\left[2,3\right.$, 4]. This means that for $\operatorname{In}_{0.98} \mathrm{Mn}_{0.02} \mathrm{Sb}$ PCs we deal with the diffusive regime of conductivity $\ell<<d$. It is also possible that a contact consists of a number of smaller size contacts connected in parallel. In this case each (small) contact is most probably in the ballistic regime of conductivity $(\ell>>d)$. Taking this possibility into account, the data was analyzed in terms of the diffusive and ballistic models. We find that for high quality transparent contacts both approaches lead to results within the scatter of the experimental data (the authors of Ref. [17] have also arrived to similar conclusions analyzing AR experiments on $\mathrm{Nd}-(\mathrm{In}, \mathrm{Mn}) \mathrm{Sb} \mathrm{PCs})$.

The total conductance $\mathrm{G}(\mathrm{V})$ is given by:

$$
G(V)=(1-P) G_{N}(V)+P G_{P}(V)
$$

where $G_{N}$ is the unpolarized conductance and $G_{P}$ is the fully spin-polarized conductance. The $\mathrm{G}_{P}(\mathrm{~V})$ is zero for energies, $\mathrm{E}<\Delta$, and for energies $|\mathrm{E}|>\Delta$ is given by 


$$
G_{P}(V)=4 \beta /\left[(1+\beta)^{2}+4 Z^{2}\right],
$$

with $\beta=(\mathrm{E}+\mathrm{i} \Gamma) /\left\{\Delta^{2}-(\mathrm{E}+\mathrm{i} \Gamma)^{2}\right\}^{1 / 2}$. Here $\Delta$ is the superconductor energy gap, the interfacial scattering strength is measured with a dimensionless parameter $\mathrm{Z}$, and a parameter $\Gamma$ is introduced to take into account a quasiparticle's finite lifetime. The physical meaning of this phenomenological parameter is the enhanced probability of inelastic scattering in the diffusive regime of conductivity (see Ref. [44] for details).

Figure 10 shows the results of fitting to the AR spectra for the conductance of the spinpolarized region of the $\mathrm{In}_{0.98} \mathrm{Mn}_{0.02} \mathrm{Sb}$ being in contact with $\mathrm{Pb}$ (see bottom inset in Fig. 9). The fitting parameters are: $\Delta=1.3 \mathrm{meV}, \mathrm{Z}=0.1, \Gamma=0.1 \mathrm{meV}$, and $\mathrm{P}=0.65$. The fits result in $\mathrm{Z}$ values close to zero (metallic contacts) and high spin polarization of the ferromagnetic region in $\mathrm{In}_{0.98} \mathrm{Mn}_{0.02} \mathrm{Sb}$.

According to the results of this section, there are a few peculiarities which definitely point to some features which should be addressed in future investigations. As already mentioned, in the standard description, the ferromagnetism of a cluster is mediated through holes in the valence band [22, 23, 24, 25] which can freely move only inside this cluster. In contrast, the surrounding matrix seems to lack freely moving holes. However, our AR spectroscopy data show that a noticeable part of $\mathrm{Mn}^{2+}$ spins still remain outside of these ferromagnetic clusters and the clustered state possesses metallic properties. Based on the results, we conclude that the spin-flip scattering on Mn ions that are outside the ferromagnetic clusters is the main source for magnetic depolarization of spin-polarized holes (this possibility was recently suggested by Csontos et al. [25]). That is why the main part of the junctions under consideration has revealed properties typical for the PCs of a conventional superconductor with nonmagnetic metal. Note, that nano-scale regions with high spin-polarization of charge carriers have been directly detected in (In,Mn)Sb by Geresdi et al. [17]. The authors also found a striking difference between the temperature dependence of the local spin polarization and of the macroscopic magnetization, and demonstrated that nano-scale clusters with magnetization close to the saturated value are present even well above the magnetic phase transition temperature.

Although we have used a polycrystalline $\operatorname{In}_{0.98} \mathrm{Mn}_{0.02} \mathrm{Sb}$ sample, the data obtained may not be the results of averaging. Due to the small contact size the contact's characteristics is measured from a singe grain. So that, the AR measurements have directly revealed that 
at low temperature sample with $2 \% \mathrm{Mn}$ is decomposed into metallic nano-scale regions with the carrier spin polarization up to $\approx 65 \%$.

\section{SUMMARY}

In this report, a narrow-gap higher mobility semiconducting alloys $\operatorname{In}_{1-x} \mathrm{Mn}_{x} \mathrm{Sb}(\mathrm{x}=0$ and $2.0 \%$ ) were synthesized in polycrystalline form. The magnetostatic measurements have been used to probe magnetic properties of these DMSs. We observed an unconventional magnetization vs temperature behavior. Namely, the magnitude of the magnetization is increased as temperature increases in temperature range $4.2 \div 300 \mathrm{~K}$. Wile we cannot at the moment rule out completely a possibility that a small number of MnSb or Mn-reach nanoclusters are formed in (InMn)Sb matrix and affect the magnetization, we believe that reentrant ferromagnetism we observe is due to ( $\mathrm{InMn}) \mathrm{Sb}$ matrix's properties. In our opinion, the results are consistent with the recently proposed models [8, 9]: for carrier-mediated ferromagnetism of DMSs, the increasing temperature leads to an increased number of thermally excited carriers which may be sufficient to increase the exchange coupling between magnetic impurities and thereby to increase the magnetization over some range of temperature. The point contact Andreev reflection spectroscopy has been used to probe charge carriers spin polarization in $\mathrm{In}_{0.98} \mathrm{Mn}_{0.02} \mathrm{Sb}$ at low temperature. Mostly, the conventional Andreev reflection with an excess current and almost doubling of the contact's normal state conductance has been detected. But in a few cases we detected the PC characteristics which can be attributed to nanoscale ferromagnetic ordered regions with spin-polarized charge carriers. For these nano-clusters a magnitude of the carrier spin polarization may be as large as up to $65 \%$. The results can be explained by the fact that at low temperature the samples are decomposed on metallic ferromagnetic regions with high current spin polarization. The findings constitute that ferromagnetism in $\operatorname{In}_{1-x} \mathrm{Mn}_{x} \mathrm{Sb}$ materials may happen to be strong. However, whether such a possibility can be realized depends on many parameters and the quest remains highly nontrivial.

The authors would like to thank V.I. Kamenev for X-ray analysis. We are also grateful to T.E. Konstantinova and V.V. Burkhoveckii for sample's EDS analysis and useful discussion. The work is partially supported by RFBR (Project \# 05-02-17666) and NASU under the 
Program 'Nanosystems, Nanomaterials, Nanotechnologies'.

[1] H. Ohno, Science 281 (1998) 951; T. Dietl, H. Ohno, Physica E 9 (2001) 185.

[2] V. A. Ivanov, T. G. Aminov, V. M. Novotortsev, V. T. Kalinnikov, Izvestiya Akad. Nauk, Ser. Khim., 11 (2004) 2255 (in Russian) [Russian Chem. Bull., Intern. Edition, 53 (2004) 2357].

[3] T. Jungwirth, J. Sinova, J. Mašek, J. Kučera, A. H. MacDonld, Rev. Mod. Phys. 78 (2006) 809.

[4] T. Dietl, J. Appl. Phys. 103 (2008) 07D111 ; T. Dietl, J. Phys.: Condens. Matter. 19 (2007) 165204.

[5] T. Jungwirth, K. Y. Wang, J. Maek, K. W. Edmonds, J. König, J. Sinova, M. Polini, N. A. Goncharuk, A. H. MacDonald, M. Sawicki, A. W. Rushforth, R. P. Campion, L. X. Zhao, C. T. Foxon, B. L. Gallagher. Phys. Rev. B 72 (2005) 165204.

[6] T. Dietl, H. Ohno, F. Matsukura, Phys. Rev. B 63 (2001) 195205.

[7] P.M. Krstajić, V.A. Ivanov, F.M. Peeters, V. Fleurov, and K. Kikoin, Europhysics Lett. 61 (2003) 235; P.M. Krstajić, F.M. Peeters, V.A. Ivanov, V. Fleurov, K. Kikoin, Phys. Rev. B 70 (2004) 195215.

[8] A. G. Petukhov, I. Žutic, S. C. Erwin, Phys. Rev. Lett. 99 (2007) 257202.

[9] M. J. Calderon, S. Das Sarma, Phys. Rev. B 75 (2007) 235203.

[10] M. Csontos, G. Mihály, B. Jankó, T. Wojtowcz, X. Liu, and J.K. Furdyna, Nature Materials, $4(2005) 447$.

[11] S. M. Ryabchenko, Y. G. Semenov, Sov. Phys. JETP 57 (1983) 825.

[12] A. Kaminski, S. Das Sarma, Phys. Rev. Lett. 88 (2002) 247202.

[13] Y. Ji, G. J. Strijkers, F. Y. Yang, C. L. Chien, J. M. Byers, A. Anguelouch, G. Xiao, A. Gupta, Phys. Rev. Lett. 86 (2001) 5585; G. J. Strijkers, Y. Ji, F. Y. Yang, C. L. Chien, and J. M. Byers, Phys. Rev. B 63 (2001) 104510; V. N. Krivoruchko, V. Yu. Tarenkov, Phys. Rev. B 78 (2008) 054522.

[14] R. J. Soulen Jr., J. M. Byers, M. S. Osofsky, B. Nadgorny, T. Ambrose, S. F. Cheng, P. R. Broussard, C. T. Tanaka, J. Nowak, J. S. Moodera, A. Barry, J. M. D. Coey, Science 282 (1998) 85.

[15] I. I. Mazin, A. A. Golubov, B. Nadgorny, J. Appl. Phys. 89 (2001) 7576; B. Nadgorny, I. I. 
Mazin, M. Osofsky, R. J. Soulen, Jr., P. Broussard, R. M. Stroud, D. J. Singh, V. G. Harris, A. Arsenov, Ya. Mukovskii, Phys. Rev. B 63 (2001) 184433; V. N. Krivoruchko, V. Yu. Tarenkov, Phys. Rev. B 75 (2007) 214508.

[16] M. J. de Jong, C.W.J. Beenakker, Phys. Rev. Lett. 74 (1995) 1657 ; I.I. Mazin, Phys. Rev. Lett. 83 (1999) 1427.

[17] A. Geresdi, A. Halbritter, M. Csontos, Sz. Csonka, G. Mihály, T. Wojtowicz, X. Liu, B. Jankó, J.K. Furdyna, Phys. Rev. B 77 (2008) 233304.

[18] R. P. Panguluri, B. Nadgorny, T. Wojtowicz, W. L. Lim, X. Liu, J. K. Furdyna, Appl. Phys. Lett. 84 (2004) 4947.

[19] T. Wojtowicz, G. Cywinski, W. L. Lim, X. Liu, M. Dorowolska, J. K. Furdyna, K.M. Yu, W. Walukiewicz, G.B. Kim, M. Cheon, X. Chen, S.M.Wang, H. Luo, Appl. Phys. Lett. 82 (2003) 4310.

[20] J. G. Braden, J.S. Parker, P. Xiong, S.H. Chun and N. Samarth, Phys. Rev. Lett. 91 (2003) 056602 ; ibid. 93 (2004) 69704 .

[21] R. P. Panguluri, K.C. Ku, T. Wojtowicz, X. Liu, J. K. Furdyna, Y. B. Lyanda-Geller, N. Samarth, B. Nadgorny, Phys. Rev. B 72 (2005) 054510.

[22] C. Timm, F. Schäfer, F. von Oppen, Phys. Rev. Lett. 89 (2002) 137201; C. Timm, J. Phys.: Conden. Matt., 15 (2003) R1865 .

[23] R.N. Bhatt, M. Berciu, M.P. Kennet, X. Wan, J. Supercond. 15 (2002) 71.

[24] G. Alvarez, E. Dagotto, Phys. Rev. B 68 (2003) 045202.

[25] M. Csontos, T. Wojtowcz, X. Liu, M. Dobrowolska, B. Jankó, J.K. Furdyna, G. Mihály, Phys. Rev. Lett. 95 (2005) 227203 .

[26] I. Vurgaftmana, J. R. Meyer, L. R. Ram-Mohan, J. Appl. Phys. 89 (2001) 5815.

[27] O. N. Pashkova, V. P. Sanygin, V. A. Ivanov, A. G. Padalko, V. M. Novotortsev, Neorganicheskie Materialy, 42 (2006) 519 (in Russian) [Inorganic Materials, 42 (2006) 459]; V. M.Novotortsev, I.S. Zakharov, A.V. Kochura, S.F. Marenkin, R. Laiho, E. Lahderanta, A. Lashul, A.G. Veresov, A.V. Molchanov, G. S. Yur'ev, Russ. J. Inorganic Chem. 51 (2006) 1627.

[28] V. A. Ivanov, O.N. Pashkova, E. A.Ugolkova, V.P. Sanygin, R.-M. Galera, Neorganicheskie Materialy, 44 (2008) 1168 (in Russian) [Inorganic Materials, 44 (2008) 1041].

[29] M. Moreno, A. Trampert, B. Jenichen, L. Däweritz, K. H. Ploog, J. Appl. Phys. 92 (2002) 
4672 .

[30] A. Sundaresan, C. N. R. Rao, Nano Today 4 (2009) 96.

[31] V.G. Storchak, , D.G. Eshchenko, H. Luetkens, E. Morenzoni, R.L. Lichti, S.F. Marenkin, O.N. Pashkova, J.H. Brewer, Physica B 374-375 (2006) 430.

[32] S. Cho, S. Choi, Gi-Beom Cha, S. C., B. J. Kim, Y. C. Kim, B.-Ch. Choi, Phys. Rev. Lett. 88 (2002) 257203; T. M. Pekareka, B. C. Crooker, I. Miotkowski, A. K. Ramdas, J. Appl. Phys. 83 (1998) 6557.

[33] A. C. Dust, R.N. Bhatt, P.A. Wolff, Phys. Rev. B 65 (2002) 235205.

[34] J. M. D. Coey, M. Venkatesan, C. B. Fitzgerald, Nat. Mater. 4 (2005) 173.

[35] N. W. Ashcroft, N. D. Mermin, Solid State Physics (Saunders, Philadelphia, 1976).

[36] Y. Yeshurun, M. B. salamon, K. V. Rao, H. S. Chen, Phys. Rev. B 24 (1536) 1981.

[37] M. Glunk, J. Daeubler, L. Dreher, S. Schwaiger, W. Schoch, R. Sauer, W. Limmer, A. Brandlmaier, S. T. B. Goennenwein, C. Bihler, M. S. Brandt, Phys. Rev. B 79 (2009) 195206.

[38] A. Savoyant, A. Stepanov, R. Kuzian, C. Deparis, C. Morhain, and K. Grasza, Phys. Rev. B 80 (2009) 115203.

[39] E. A. Pashitskii, S. M. Ryabchenko, Sov. Phys. Solid State 21 (1979) 322.

[40] K. Kubo,N. Ohata, J. Phys. Soc. Jpn. 33 (1972) 21.

[41] E. L. Wolf, Principles of Electron Tunneling Spectroscopy (Oxford University Press. New York, 1985).

[42] K. Seeger, Semiconductor Physics (Springer - Verlag, Wien New York, 1973).

[43] G. Wexler, Proc. Phys. Soc. London 89, 927 (1966); Yu. V. Sharvin, JETP 21 (1965) 655.

[44] A. Plecenik, M. Grajcar, Š. Beňačka, P. Seidel, A. Pfuch, Phys. Rev. B 49 (1994) 10016.

\section{Figure Captions}

FIG. 1. SEM image of $\operatorname{In}_{0.98} \mathrm{Mn}_{0.02} \mathrm{Sb}$ sample. For numbered points the results of the chemical composition analysis are summarized in Table I.

FIG. 2. (Color online) Field dependence of $\operatorname{In}_{0.98} \mathrm{Mn}_{0.02} \mathrm{Sb}$ bulk sample magnetization; $\mathrm{T}$ $=4.2 \mathrm{~K}$ (black), $\mathrm{T}=77.3 \mathrm{~K}$ (blue) and $\mathrm{T}=300 \mathrm{~K}$ (red) curves. Upper inset: temperature dependence of $\operatorname{~nn}_{0.98} \mathrm{Mn}_{0.02} \mathrm{Sb}$ magnetization at $\mathrm{H}=1 \mathrm{kOe}$. Bottom inset: field dependence of MnSb sample magnetization; $\mathrm{T}=300 \mathrm{~K}$. 
FIG. 3. (Color online) Low magnetic field magnetization dependence in $\operatorname{In}_{0.972} \mathrm{Mn}_{0.028} \mathrm{Sb}$ $(\mathrm{x}=0.028)$ epitaxial film (open circles) [18] and in $\mathrm{In}_{0.98} \mathrm{Mn}_{0.02}$ Sb bulk sample.

FIG. 4. Schematic diagram of the electronic structure in a hole-doped DMS.

FIG. 5. (Color online) Temperature dependence of the average total magnetization per ion $M(T)=m(T)+\frac{N_{h}}{N_{i}} s(T)$, showing reentrant behavior at $\mathrm{T}=\mathrm{T}_{C 2}$ as temperature is raised. Inset: temperature dependence of the average spin of conduction electron $\mathrm{s}(\mathrm{T})$ and spin of impurity ion $\mathrm{m}(\mathrm{T})$. For material's parameters see text.

FIG. 6. Low-temperature dependence of the $\operatorname{In}_{0.98} \mathrm{Mn}_{0.02} \mathrm{Sb}$ resistivity; the curve is normalized on $\rho_{(\operatorname{InMn}) S b}(25 K)=4.09 \times 10^{-4} \Omega \cdot \mathrm{cm}$. Inset: comparative temperature dependences of the resistivity for $\mathrm{In}_{0.98} \mathrm{Mn}_{0.02} \mathrm{Sb}$ and $\mathrm{InSb}$; the curves are normalized on $\rho_{\text {InSb }}(275 K)=2.24 \times 10^{-2} \Omega \cdot \mathrm{cm}$.

FIG. 7. Experimental density of state near the Fermi $\varepsilon_{F}$ of $\operatorname{InSb}$ and $\operatorname{In}_{0.98} \mathrm{Mn}_{0.02} \mathrm{Sb}$ at $\mathrm{T}=4.2 \mathrm{~K}$.

FIG. 8. Typical current-voltage (I-V) dependence of the $\mathrm{Pb}-\mathrm{In}_{0.98} \mathrm{Mn}_{0.02} \mathrm{Sb}$ contact at $\mathrm{T}=4.2 \mathrm{~K}$. Upper inset: low temperature region dependence of the contact's resistance $\mathrm{R}(\mathrm{T})$. Bottom inset: the contact's Andreev reflection spectra at $\mathrm{T}=4.2 \mathrm{~K}$.

FIG. 9. An example of the current-voltage (I-V) dependence of the $\mathrm{Pb}-\mathrm{In}_{0.98} \mathrm{Mn}_{0.02} \mathrm{Sb}$ contact at $\mathrm{T}=4.2 \mathrm{~K}$ in the case when $\mathrm{S}$ tip is in contact with ferromagnetic ordered nanoregion of $\operatorname{In}_{0.98} \mathrm{Mn}_{0.02} \mathrm{Sb}$. Top inset: low temperature region dependence of the contact's resistance $R(T)$. Bottom inset: the contact's Andreev reflection spectra at $T=4.2 \mathrm{~K}$.

FIG. 10. Fits to modified theory (see text) for the conductance spectrum of $\mathrm{Pb}-$ $\mathrm{In}_{0.98} \mathrm{Mn}_{0.02} \mathrm{Sb}$ point contact at $\mathrm{T}=4.2 \mathrm{~K}$; fitting parameters are $\Delta=1.3 \mathrm{meV}, \mathrm{Z}=0.1, \Gamma$ $=0.1 \mathrm{meV}$, and $\mathrm{P}=0.65$. 
Table I. EDS data of the $\operatorname{In}_{0.98} \mathrm{Mn}_{0.02}$ Sb sample chemical composition.

\begin{tabular}{|c|c|c|c|c|}
\hline point $\backslash$ ion & Mn & In & Sb & O \\
\hline 1 & $\mathbf{1 . 1 7}$ & 46.65 & 50.73 & 1.46 \\
\hline 2 & $\mathbf{2 . 4 4}$ & 43.64 & 52.53 & 1.40 \\
\hline 3 & $\mathbf{1 . 5 0}$ & 45.67 & 51.22 & 1.61 \\
\hline 4 & $\mathbf{1 . 6 7}$ & 45.84 & 50.07 & 2.42 \\
\hline 5 & $\mathbf{3 . 3 9}$ & 44.11 & 51.96 & 0.54 \\
\hline 6 & $\mathbf{1 . 5 0}$ & 47.29 & 50.49 & 0.72 \\
\hline 7 & $\mathbf{1 . 6 9}$ & 45.89 & 50.99 & 1.43 \\
\hline 8 & $\mathbf{1 . 8 3}$ & 45.74 & 51.54 & 0.89 \\
\hline 9 & $\mathbf{2 . 7 8}$ & 42.69 & 51.33 & 3.20 \\
\hline 10 & $\mathbf{1 . 3 3}$ & 45.57 & 52.08 & 1.02 \\
\hline 11 & $\mathbf{1 . 9 6}$ & 45.60 & 50.81 & 1.62 \\
\hline average & $\mathbf{1 . 9 6}$ & 43.91 & 51.68 & 2.45 \\
\hline standard deviation & $\mathbf{0 . 8 0}$ & 5.78 & 1.99 & 3.44 \\
\hline
\end{tabular}




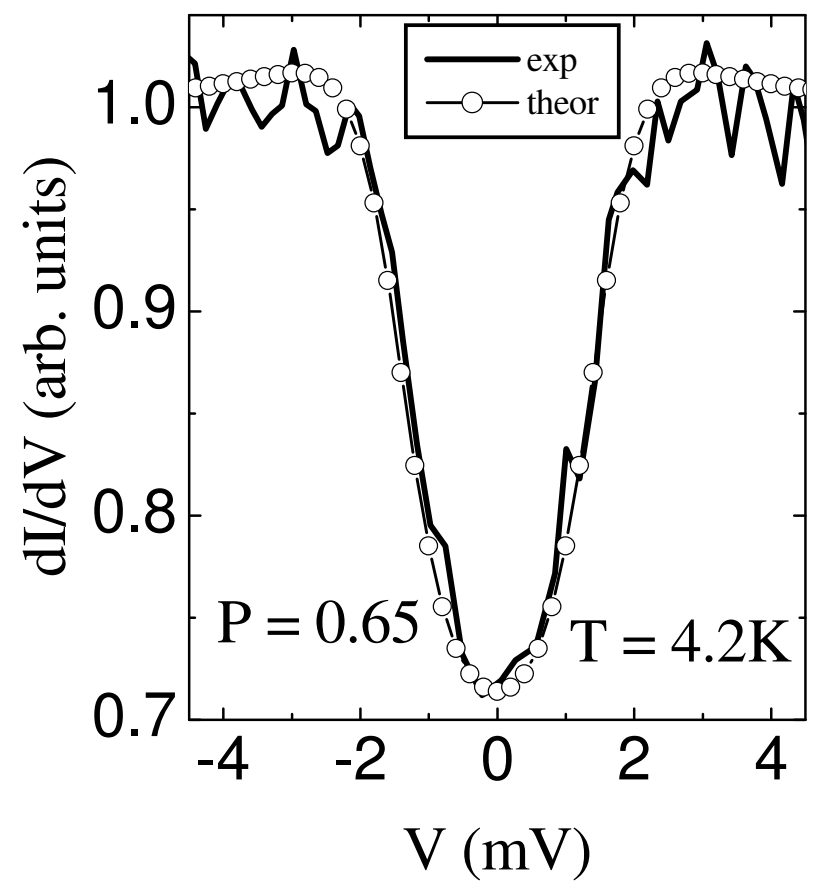

Fig. 10 


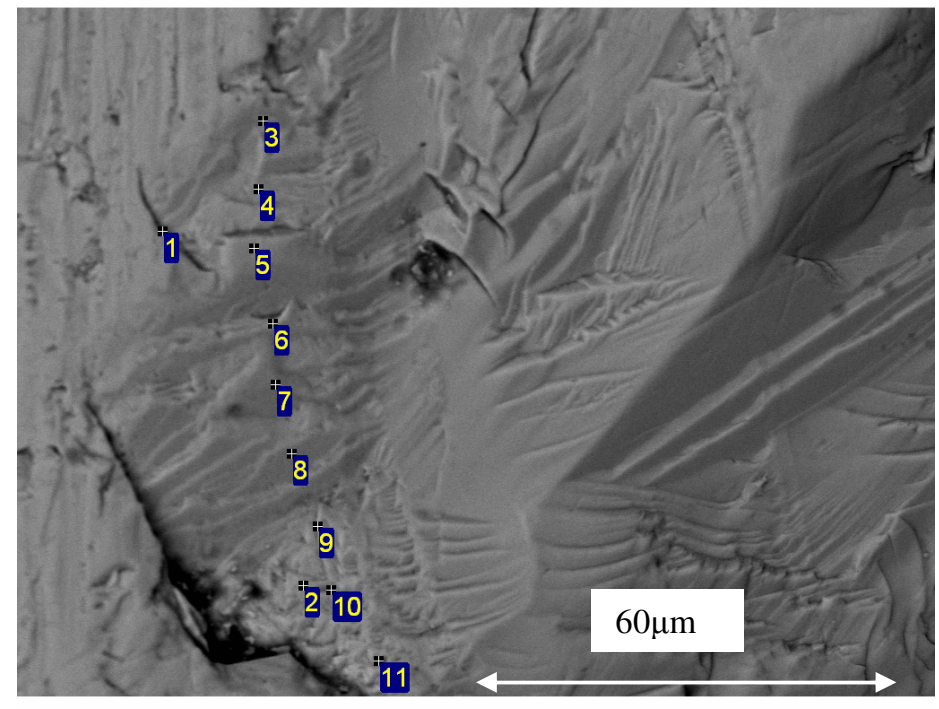

Fig. 1 


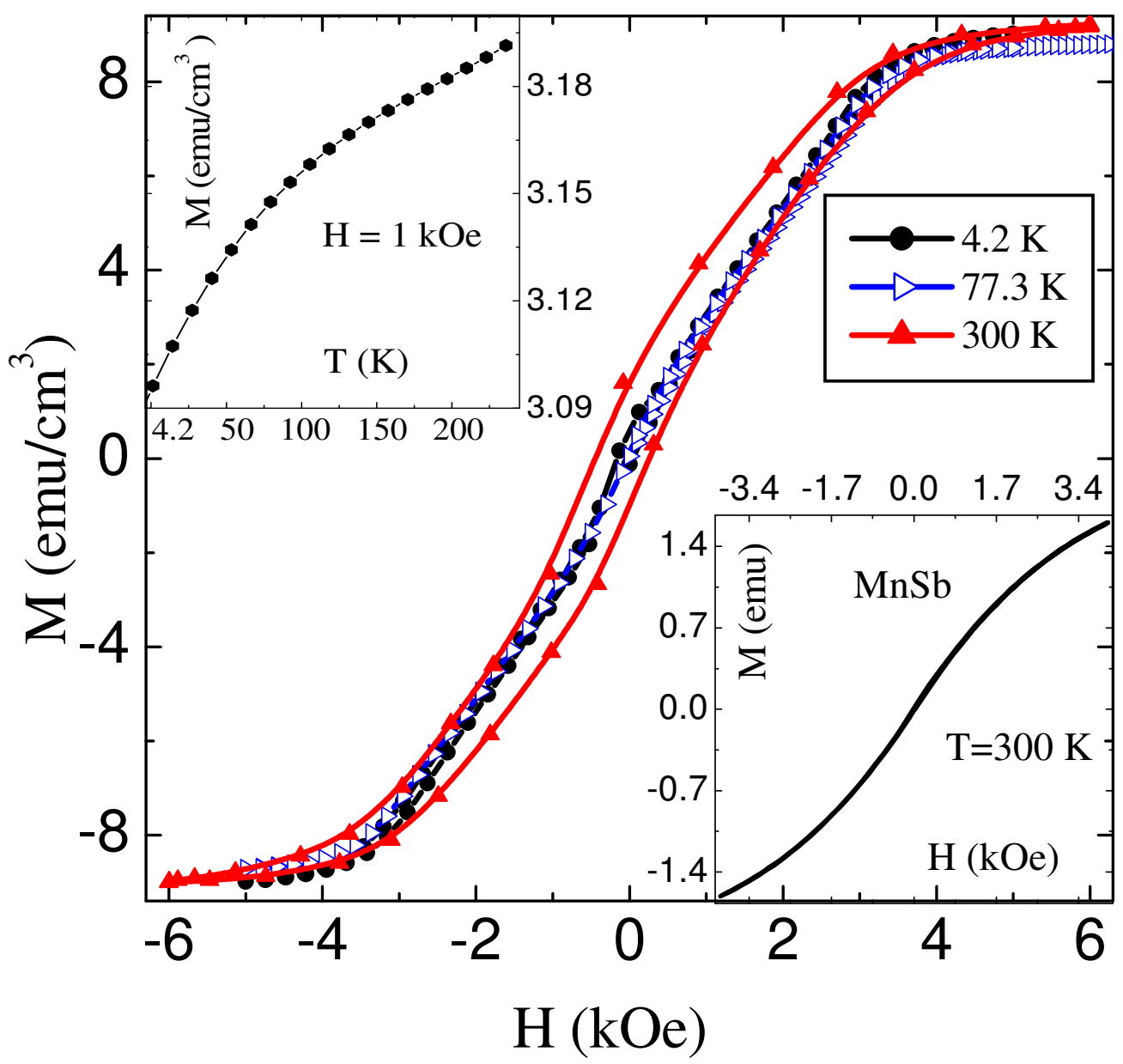

Fig. 2 


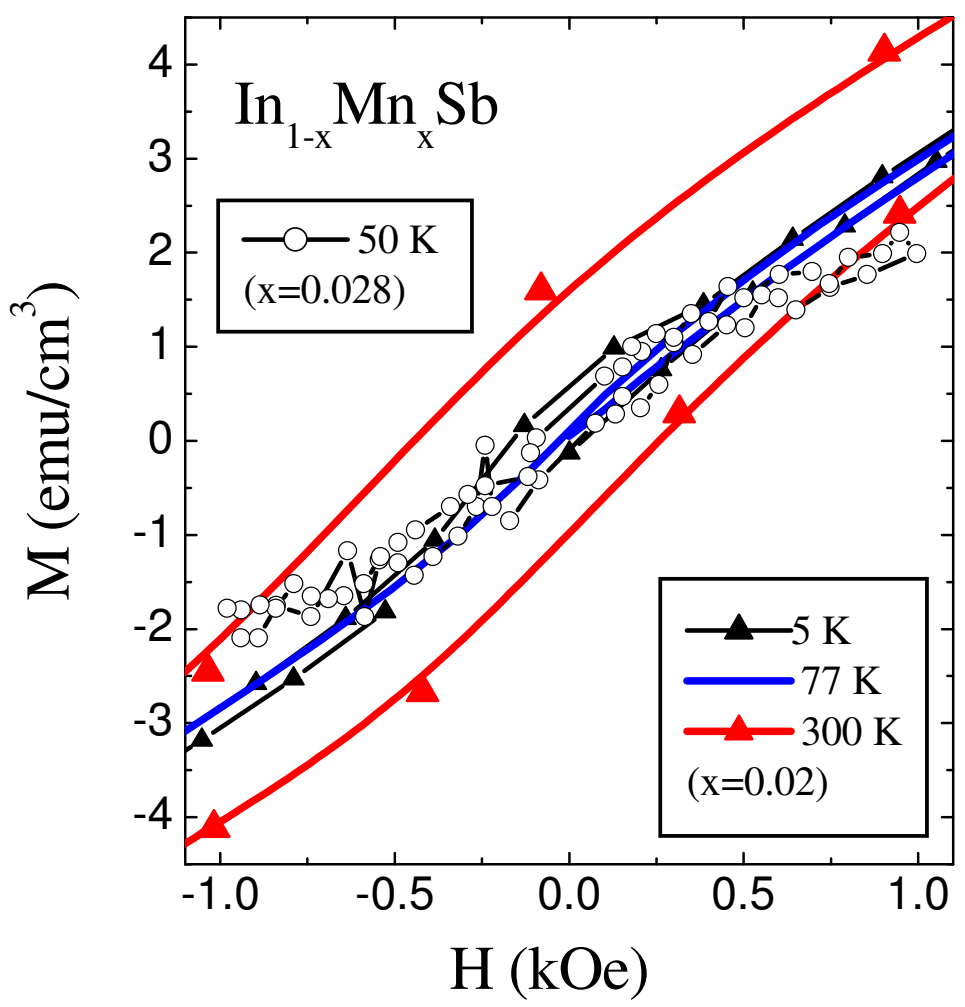

Fig. 3 


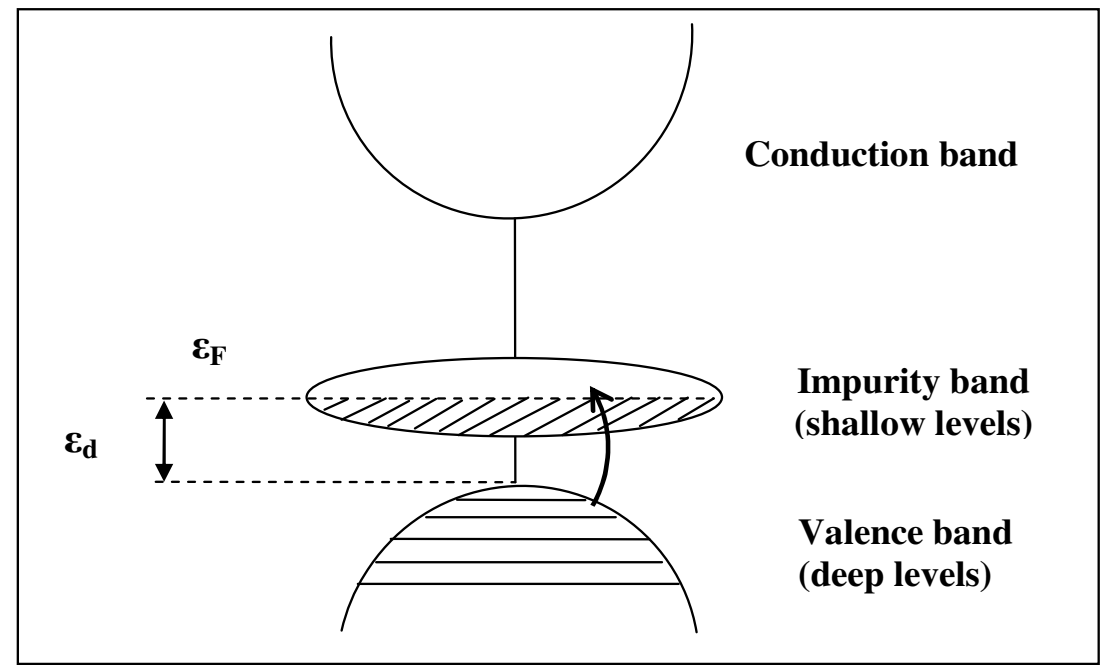

Fig. 4 


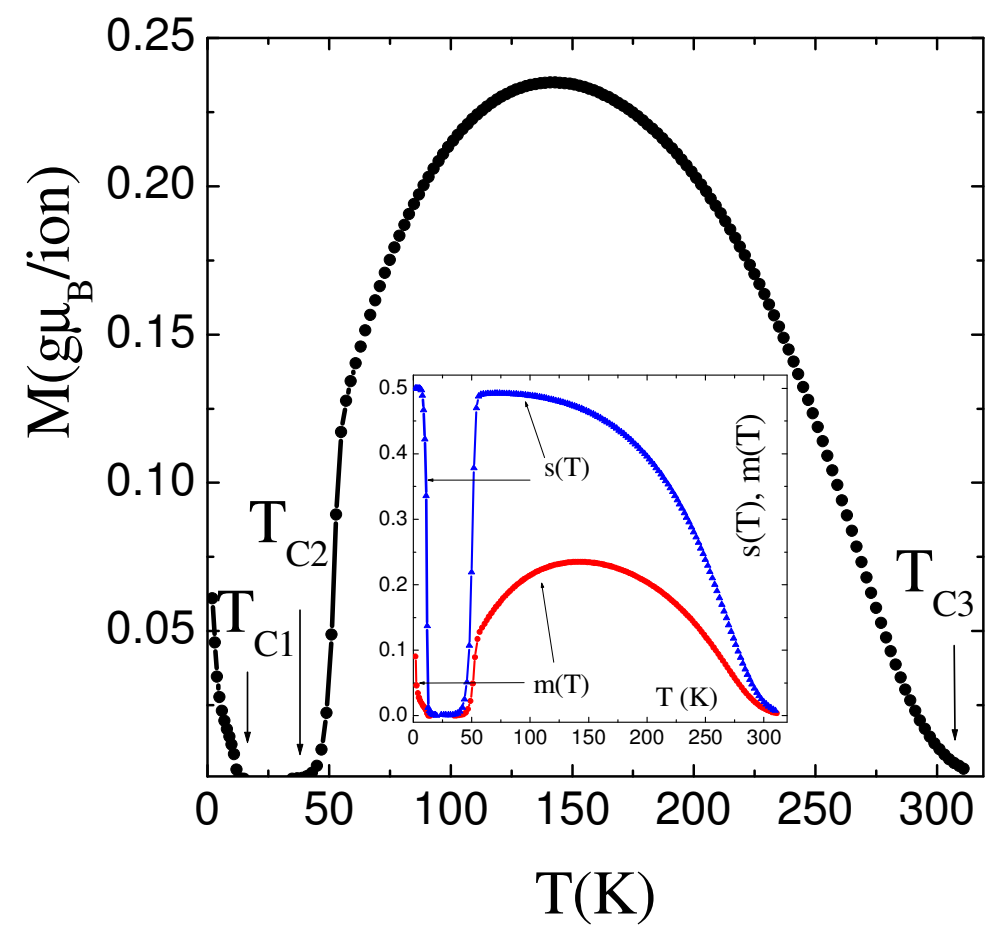

Fig. 5 


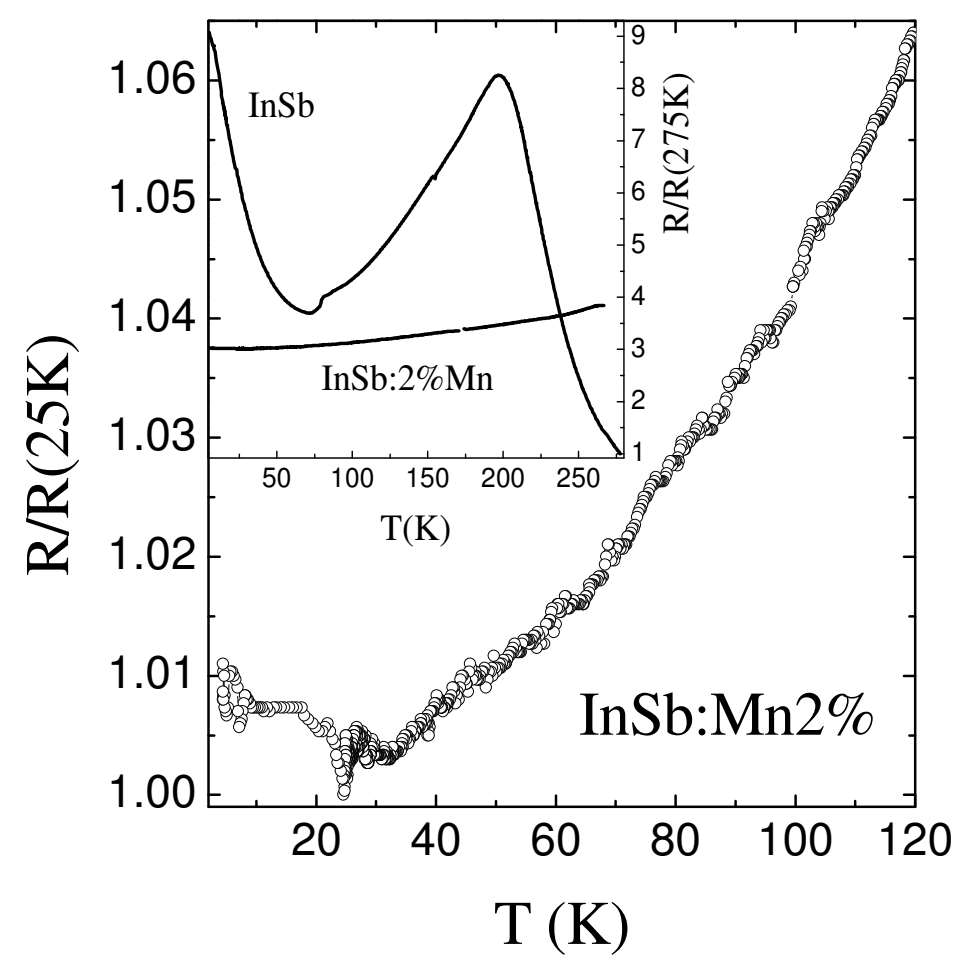

Fig. 6 


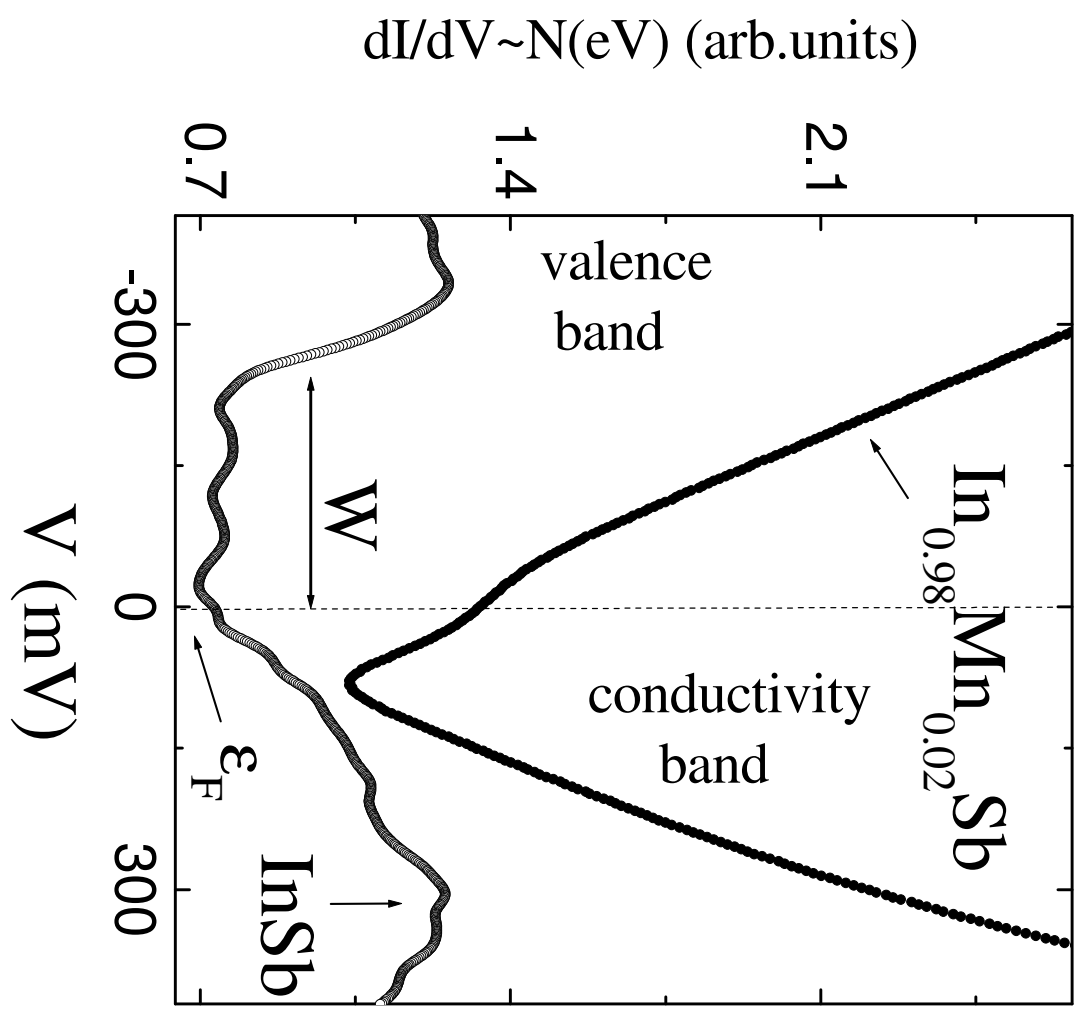




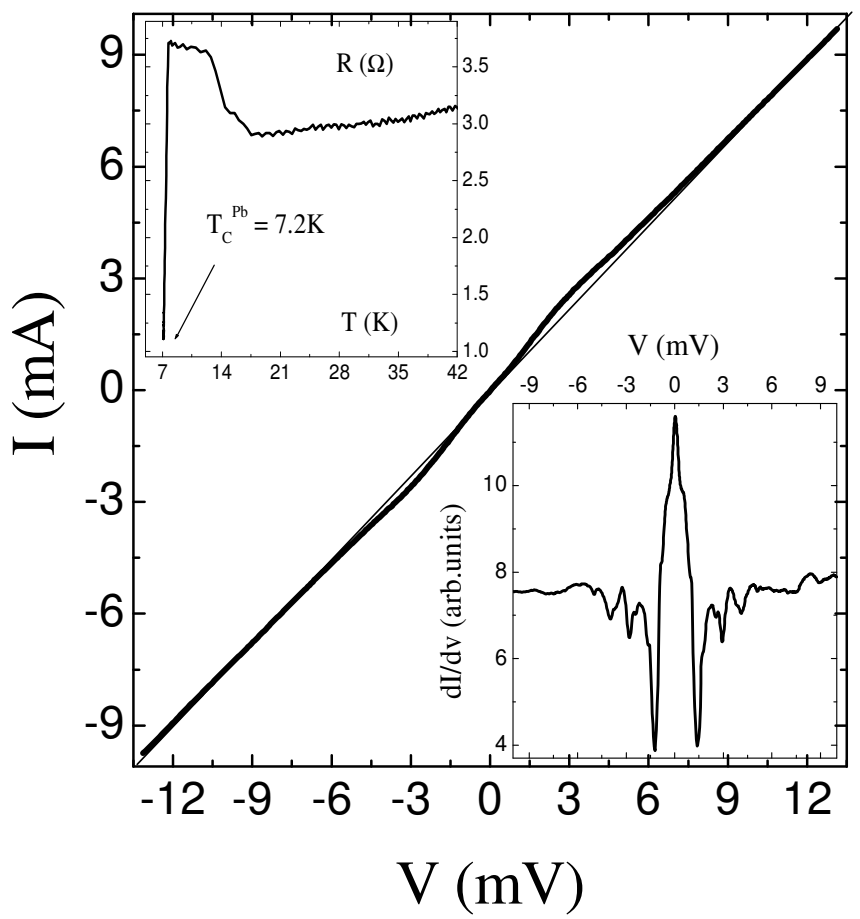

Fig. 8 


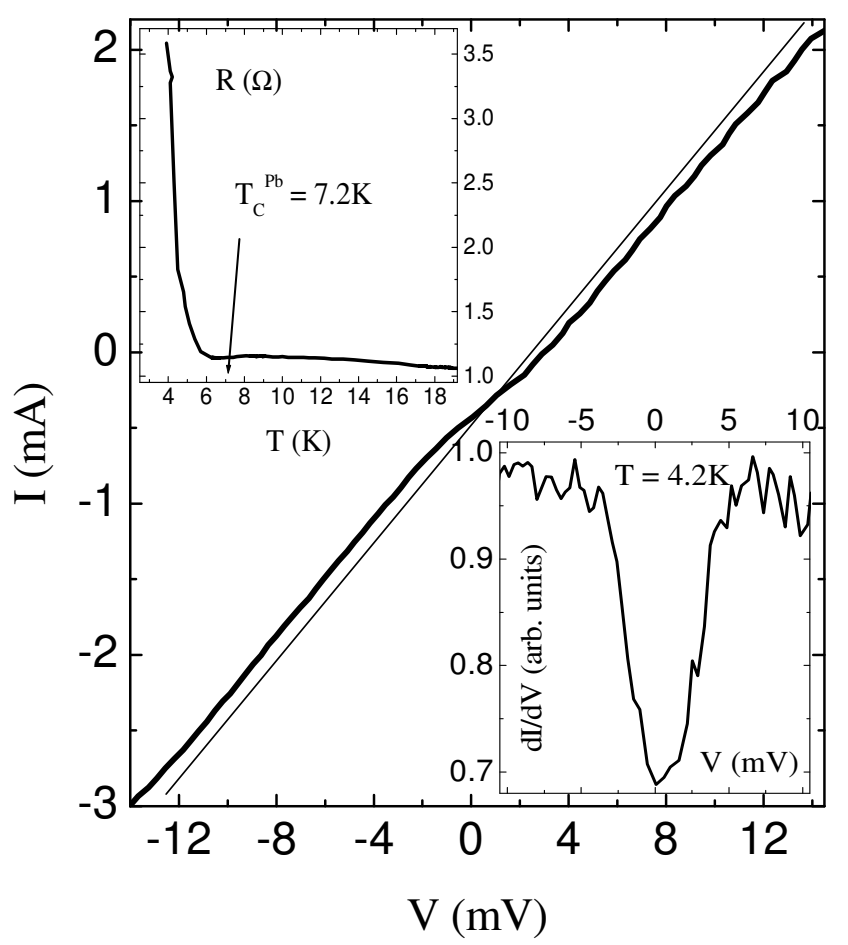

Fig. 9 